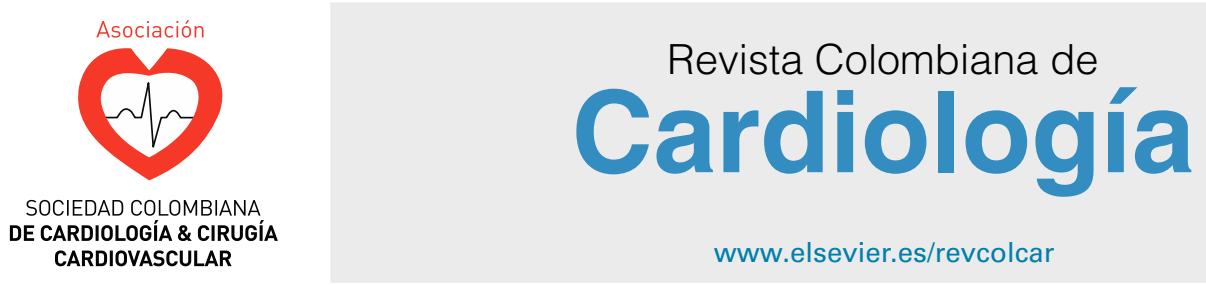

CARTA AL EDITOR

\section{Conclusiones imprecisas respecto a la confiabilidad de la Florida Patient Acceptance Survey}

\section{Indeterminate conclusions as regards the reliability of the Florida Patient Acceptance Survey}

\section{Señor Editor}

En un estudio reciente ${ }^{1}$ se obtienen evidencias psicométricas del Florida Patient Acceptance Survey, FPAS ${ }^{2}$, una medida útil para la investigación científica y la práctica clínica. No obstante, existen imprecisiones en sus conclusiones de la confiabilidad, que pueden conducir a un insostenible optimismo sobre su uso. La presente carta señala estos puntos y presenta respaldo analítico para fundamentarlos mediante varios métodos estadísticos, que incluyen una prueba de contraste para dos coeficientes alfa independientes, magnitud de la diferencia entre ambos coeficientes e intervalos de confianza.

Los autores obtienen una confiabilidad del puntaje total igual a $0.724(n=126)$, y declaran cualitativamente que este alcanza un nivel aceptable de acuerdo con el criterio de George y Mallery; también afirman que este resultado es similar al estudio original $(a l f a=0.83)$. Sin embargo, con la aplicación del método de Feldt ${ }^{3}$ para contrastar estadísticamente ambas estimaciones se halló que existe una diferencia estadísticamente significativa $\left(\chi^{2}=8.743, \mathrm{gl}=1, p\right.$ 0.003). Este resultado contrasta seriamente con la afirmación de similaridad que los autores hacen. Para detallar más esta discrepancia se puede calcular el tamaño de esta diferencia. En la métrica de diferencias estandarizadas ${ }^{4}$, entre ambas hay una distancia de 0.331 (método Lui-Weng ${ }^{5}$ ), lo que puede sugerir una diferencia pequeña ${ }^{4}$; pero dado que el nivel de confiabilidad es apenas aceptable y el impacto en la precisión del puntaje es clínicamente importante, la diferencia entre ambas puede nominarse como grande, y el coeficiente alfa del estudio de Castillo es inferior, no similar, al estudio original.

En segundo lugar, los autores concluyen que la confiabilidad es aceptable, pero la variabilidad muestral del coeficiente calculado no asegura que esta conclusión sea correcta. Para corroborarlo se deben estimar intervalos de confianza para el coeficiente alfa obtenido ${ }^{6}$. Por medio del método de Bonnet ${ }^{7}$ y con un nivel de confianza del $95 \%$, la magnitud poblacional del coeficiente va de 0.643 a 0.786 . Esta amplia banda de variabilidad está influenciada por el tamaño muestral y el nivel de confianza elegido ${ }^{6}$, y en el presente análisis incluye al nivel cuestionable y aceptable (George y Mallery); en otras palabras, el parámetro de la confiabilidad puede ser cuestionable o aceptable desde el punto de vista poblacional.

Una complicación de usar criterios "plug-and-play" (listos para usar) como el de Gregory y Mallery, es que fueron creados como una heurística sugestiva, estructurados con el juicio de los autores y no con referencias metaanalíticas empíricas. Por lo tanto, no puede considerarse aplicable a toda situación de medición, a menos que sea explícita una justificación fuertemente razonable.

Esta carta no desmerece el esfuerzo de los autores ni presenta argumentos para invalidar los análisis realizados, sino enfatiza que las conclusiones sobre la confiabilidad de los puntajes del FPAS deben lograr un mejor respaldo, específicamente de tipo empírico. De este modo, los usuarios finales pueden tomar decisiones informadas y alineadas en las prácticas basadas en la evidencia. Respecto a la adaptación del FPAS concluimos que la confiabilidad de su puntaje total es moderadamente menor que la el estudio original, y puede ser de nivel cuestionable en la población de referencia. Futuros estudios deben orientarse al incremento del tamaño muestral, así como a elegir criterios cualitativos de confiabilidad con base empírica o sintetizados de la literatura psicométrica.

\section{Financiación}

Ninguna.

\section{Conflicto de intereses}

Ninguno.

\section{Bibliografía}

1. Castillo-Sierra D, González-Consuegra R, OlayaSánchez A. Validez y confiabilidad del cuestionario Florida versión en español. Rev Colomb Cardiol. 2018;25:131-7. Disponible en https://doi.org/10.1016/j.rccar. 2017.12.018.

2. Burns JL, Serber ER, Keim S, Sears SF. Measuring patient acceptance of implantable cardiac device therapy. J Cardiovasc 
Electrophysiol. 2005;16:384-90. Disponible en http://doi.wiley. com/10.1046/j.1540-8167.2005.40134.x.

3. Feldt LS, Woodruff DJ, Salih FA. Statistical inference for coefficient alpha. Appl Psychol Meas. 1987;11:93-103. Disponible en https://doi.org/10.1177/014662168701100107.

4. Cohen J. Statistical power analysis for the behavioral sciences. $2^{\text {nd }}$. ed. Hillsdale. NJ: Erlbaum;. 1988.

5. Liu HY, Weng LJ. An effect size index for comparing two independent alpha coefficients. Brit J Math Stat Psy. 2009;62:385-400. Disponible en https://doi.org/10.1348/000711008X315518.

6. Domínguez-Lara SA, Merino-Soto M. ¿Por qué es importante reportar los intervalos de confianza del coeficiente alfa de Cronbach? Revista Latinoamericana de Ciencias Sociales. Niñez y Juventud. 2015;13:1326-8.
7. Bonett DG. Sample size requirements for testing and estimating coefficient alpha. J Educ Behav Sta. 2002;27:335-40. Disponible en https://doi.org/10.3102/10769986027004335.

César Merino-Soto ${ }^{\mathrm{a}, *}$ y Marisol Angulo-Ramos ${ }^{\mathrm{b}}$

a Universidad de San Martín de Porres, Lima, Perú

b Universidad Los Ángeles de Chimbote, Chimbote, Perú

* Autor para correspondencia.

Correos electrónicos: sikayax@yahoo.com.ar, cmerinos@usmp.pe (C. Merino-Soto).

Disponible en Internet el 27 de abril de 2020 